Article - Human and Animal Health

\title{
The Psychophysiological Responses of the Chronic Ischemic Stroke Patients to the Acute Stress were Changed
}

Omid Mirzaee ${ }^{1}$

https://orcid.org/0000-0001-7532-8535

Mojtaba Saneian ${ }^{2}$

https://orcid.org/0000-0003-4940-6899

Javad Rasouli Vani ${ }^{3}$

https://orcid.org/0000-0002-0225-2565

Kamyab Shahrivar ${ }^{2}$

https://orcid.org/0000-0003-4825-3958

Mahmoodreza Peyravi ${ }^{1}$

https://orcid.org/0000-0001-8111-3231

Abdolhamid Shariat ${ }^{4 *}$

https://orcid.org/0000-0003-3168-9800

Boshra Hatef ${ }^{\star}$

https://orcid.org/0000-0002-2638-3463

${ }^{1}$ Shiraz University of Medical Sciences, Shiraz, Iran; ${ }^{2}$ Shiraz University of Medical Sciences, Student research committee, Shiraz, Iran; ${ }^{3}$ Baqiyatallah University of Medical Sciences, Department of Biochemistry, School of Medicine, Tehran, Iran; ${ }^{4}$ Shiraz University of Medical Sciences, Clinical Neurology Research Center, Shiraz, Iran; ${ }^{5}$ Baqiyatallah university of medical science, Neuroscience research center, Tehran, Iran.

Received: 2018.09.13; Accepted: 2019.07.08.

${ }^{*}$ Correspondence: boshrahatef@bmsu.ac.ir; Tel.: +98-21-88040060 (B.H.)

*Correspondence: Shariat123@gmail.com; Tel.: +98-21-88040060 (A.S.)

\section{HIGHLIGHTS}

- The physiological response of ischemic stroke to acute stress (TSST) was assessed

- Patients had higher baseline cortisol level and anxiety score than health subjects

- The level of cortisol did not increase in the patients after TSST

- The stress increased the relative low frequency of HRV in both groups

- Alpha 1 of DFA of HRV of patients was significantly more than health subjects 
Abstract: Mood disorder is one of the complications of stroke. The inability to cope with stress is also a prognosis of depression and anxiety. The aim of this study is to assess the response of stress system in the post stroke patients. Twelve healthy controls $(\mathrm{HC})$ and twelve post-stroke patients after filling in the State-Trait Anxiety Inventory completed the Trier Social Stress Test (TSST) which induces acute stress. Salivary samples were collected to determine salivary cortisol levels and ECG record were taken in four times (before, right after stress, after two recoveries: 20 and 40 minutes after stress). ECG was also recorded during TSST and then the linear and non-linear features of Heart Rate Variability (HRV) were analyzed. The results showed that trait anxiety score and baseline salivary cortisol level were higher in post stroke than HC group ( $P$-value $<0.05)$. The increase of cortisol level after stress was only observed in $\mathrm{HC}$ that returned to baseline after the second recovery time. The stress increased the relative low frequency of HRV in both groups, however it was significantly lower in the stroke than HC group ( $P$-value $<0.005)$. There was also a significant difference between alpha 1 DFA measures in stroke group and $\mathrm{HC}$ group ( $\mathrm{P}$-value $<0.05$ ). It is concluded that the impairment of the hormonal axis of stress system in the post-stroke patients that until now was not reported.

Keywords: ischemic stroke; acute social stress; cortisol; heart rate variation.

\section{INTRODUCTION}

All over the world, stroke is the second mortality cause so that 10 percent of 55 million deaths are due to the stroke ${ }^{1,2}$, although it is the third mortality cause in the US ${ }^{3}$. From 1990 to 2010 , the age-standardized incidence of stroke increased by $12 \%$ in low-income and middle-income countries ${ }^{4}$. In Iran, the annual stroke incidence ranged from 23 to 103 per 100,000 population ${ }^{5}$ while another study showed that the mortality rate among stroke patients is $12.1 \%{ }^{6}$. As stroke is becoming an increasing health problem, its complications must be getting more attention ${ }^{7,8}$. The most debilitating complications of stroke are psychiatric complications ${ }^{9}$. Depression, anxiety and adjustment disorder are common mood disorders after stroke ${ }^{10,11 .}$

In the general population, it is highly accepted that mood disorders is closely correlated with chronic stress ${ }^{12-14}$ and adults with mood disorder report more physiological responses to the stressful life events ${ }^{12}$. Physiologically, stress stimulates amygdala-prefrontal axis in the brain and, finally, following the excitation of Paraventricular Nucleus of Hypothalamus, Hypothalamic Pituitary Adrenocortical Axis (HPA) as well as Sympathetic Adrenal Medullary axis (SAM) will be activated ${ }^{15}, 16$. The activation of HPA axis and SAM system is an adaptive process that enables the human body to maintain physiological stability in response to stress ${ }^{17}$. In addition, the activation of HPA axis eventually causes cortisol release and SAM system activation causes the increase of heart rate and blood pressure. Therefore, Heart Rate Variability (HRV) indices can be used to evaluate the autonomic nervous system activation ${ }^{18}$.

Given the fact that ischemic stroke, as a huge source of stress, results in some changes in the autonomic nervous system, measured by HRV ${ }^{19}$, and also it affects HPA axis for the reasons specific to stroke ${ }^{20-22}$, we hypothesized that the physiologic responses to the psychosocial stress, like cortisol release and heart rate variability, might be different between the stroke patients and healthy individuals. Besides that, these altered physiological responses to stress in ischemic stroke patients may be one of the major etiologies for post-stroke mood disorder.

The aim of this study was to compare the cortisol level and heart rate variability facing acute stress, induced through Trier Social Stress Test (TSST), and also to compare physiologic biomarkers of chronic stress between patients with chronic stroke and healthy controls that until now was not studied. 


\section{MATERIAL AND METHODS}

\section{Participants}

Twenty-two male individuals entered the study (12 healthy controls and 12 post- stroke patients). The inclusion criteria were age, 18 up to 70 years old, ischemic stroke that happened $>1$ year before the study and is approved by computerized tomography (CT) scan or Magnetic Resonance Imaging (MRI)., Modified Ranking Score $<=3$ (in post- stroke patients), no history of other systemic diseases like diabetes, cardiovascular disease, autoimmune diseases and psychiatric diseases, no regular neurophysiologic medication uptake, no smoking at least one day before the study and no heavy exercise at least one month before the study. The health control $(\mathrm{HC})$ group was matched in terms of age, gender and body mass index with patients group. All participants completed and signed the informed consent before their participation in the study. The number of clinical trial assignment is IRCT20171128037666N1.

\section{Procedure}

At the arrival, the participants were given a demographic questionnaire and State-Trait Anxiety Inventory. After filling in these questionnaires, the individuals were asked to wash their mouth and after a minute give a salivary sample. After that, a blood sample was taken. Then, they rested for 30 minutes. As the level of cortisol and heart rate variability are dependent on circadian rhythm, all the subjects were tested at the same time (between 10:00 AM to 3:00 PM that the changes of cortisol is minimum) and the same place. Salivary samples and electrocardiography (ECG) record were taken in four times (before TSST, right after TSST, after two recoveries: 20 and 40 minutes after TSST). ECG was also recorded during TSST. TSST protocol started with a 5-minute speech preparation before coming to the test room and proceeded with a 3-minute speech performance (an explanation of the job and family status) against two examiners and then completed by a 12-minute math portion. In the math portion, individuals were asked to sequentially subtract the number 13 from 1022 to the end. If the participant made a mistake, the examiners would notice them with the following sentence: "That is incorrect, please start over from 1022". The subjects stayed in standing position during the test ${ }^{23}$. The psychological report of anxiety by emotional visual analogue scale (EVAS) from most calm to most anxious was also taken at 4 times of the test (rest, after stress, after first and second recovery times).

\section{Salivary cortisol measurement}

The participants were asked to have enough sleep last night, not eat anything one hour before test and not have clear stress in the time of test. If they did not adhere these conditions, the test was done in the next visit. The saliva samples were obtained minimally $(0.5 \mathrm{~mL})$ and then transferred to a refrigerator kept at the temperature of -80 Celsius degree. The human saliva cortisol enzyme immunoassay (EIA) kit from ZellBio Company made in German was used with the procedure been done based on kit's instruction. The negative curve of TDS was determined and then the level of cortisol according to OD adjusted on the curve was assessed in each sample.

\section{Heart rate recording}

The heart rate was recorded with ECG instrument designed by Biomed Company from Iran. One electrode was attached to the left midclavicular line above the heart position, and the second electrode was attached to the left sternal border below the heart position. The third electrode was attached to the right lower quadrant of the abdomen. The subjects stayed in sitting position without deep breathing or speaking during the test. Data was saved for 2 minutes in each section (except during TSST, which lasted for 15 minutes) and relayed to an analog-to-digital converter in the sampling rate of $256 \mathrm{~Hz}$. The HRV was analyzed with MATLAB software, Heart Rate Variability analysis codes. The linear features extracted from $R R$ series in the time domain were mean and SD of RR. In the frequency domain, High 
Frequency (HF) power (0.15- $0.5 \mathrm{~Hz})$, Low Frequency (LF) power $(0.05-0.15 \mathrm{~Hz})$, very LF power $(0-0.04 \mathrm{~Hz})$ and finally the ratio of LF/HF components were analyzed 24 . The non-linear features extracted in time domain were SD1 and SD2 of Poincaré Plot ${ }^{25}$, the alpha 1 of Detrended Fluctuation Analysis (DFA) ${ }^{26}$, sample entropy and, in the frequency domain, spectral entropy (SpeEn) ${ }^{27}$.

\section{Statistics}

The t-test was used to compare age, BMI, plasma factors, the score of spilberger's questioners, cortisol and heart rate between two groups. The chi-squared test was also used to compare the prevalence of education level between groups. To compare the cortisol level and HRV features between the sections of the study and two groups, two-way mixed model ANOVA with following related tests were used. The $p$-value less than 0.05 was considered as significance.

\section{RESULTS}

Twelve male post-stroke patients and twelve male healthy controls recruited in this study (mean age 56.5 in stroke group and 51.6 in healthy individuals, $P$-value $<0.05$ ). Table 1 shows the comparison of demographic characteristics and biomarkers between stroke group and healthy control group. There was no significant difference in BMI and education between two groups, however trait anxiety score and baseline salivary cortisol level were significantly higher in stroke group (P-value< 0.05). Moreover, we analyzed some biomarkers (the plasma malonaldehyde as a stress oxidative enzyme and plasma glutathione and catalase as the antioxidant enzymes) as the secondary findings but there was no significant difference in biomarker levels between two groups. The result of psychological report of anxiety by emotional visual analogue scale (EVAS) showed that anxiety increased after stress in both groups and returned to baseline after recovery $(F=176.15, P$-value $<0.00001)$. The level of EVAS was higher in the stroke than HC group $(\mathrm{f}=4.39, \mathrm{P}$-value $<0.05)$ (figure 1 ). 
Table 1: the demographic and biochemical markers of two groups and result of comparisons between them.

\begin{tabular}{|c|c|c|c|}
\hline & $\begin{array}{l}\text { Healthy controls } \\
\text { G. }\end{array}$ & $\begin{array}{l}\text { Ischemic Stroke } \\
\text { G. }\end{array}$ & $\begin{array}{l}\text { P-valu } \\
\text { e }\end{array}$ \\
\hline Age(years) & $51.6 \pm 3$ & $56.5 \pm 6$ & 0.052 \\
\hline BMI(weigh/Heigth² $)$ & $26 \pm 4$ & $25 \pm 4$ & 0.48 \\
\hline $\begin{array}{l}\text { Education(\%Diploma and under } \\
\text { Diploma) }\end{array}$ & 76.9 & 92.3 & 0.29 \\
\hline Employed (\%) & 63.6 & 58.3 & 0.56 \\
\hline Modified Rankin Scale for & & & \\
\hline $\begin{array}{ll}\text { Neurologic } & \text { Disability }(\% \\
\text { slight/moderate) } & \end{array}$ & - & $75 / 25$ & - \\
\hline Trait anxiety score & $41.38 \pm 5.6$ & $46.38 \pm 5.2$ & 0.027 \\
\hline State anxiety score & $36.69 \pm 6.5$ & $36.69 \pm 6.7$ & 1 \\
\hline $\begin{array}{l}\text { Side of Stroke } \\
\text { (\% Both/Right/Left) }\end{array}$ & - & $46.2 / 30.8 / 23.1$ & \\
\hline Plasma malonaldehyde $(\mu \mathrm{mol} / \mathrm{L})$ & $0.0061 \pm 0.0005$ & $0.0064 \pm 0.0005$ & 0.19 \\
\hline Plasma Glutathione(mM) & $3.48 \pm 0.4$ & $2.96 \pm 1.2$ & 0.19 \\
\hline Plasma Catalase (mM) & $3.59 \pm 2.4$ & $4.87 \pm 2.2$ & 0.2 \\
\hline Salivary cortisol (ng/ml) & $19.2 \pm 13$ & $43.5 \pm 28$ & 0.012 \\
\hline Heart Rate(beats/minute) & $84.52 \pm 8$ & $85.2 \pm 11.5$ & 0.89 \\
\hline
\end{tabular}

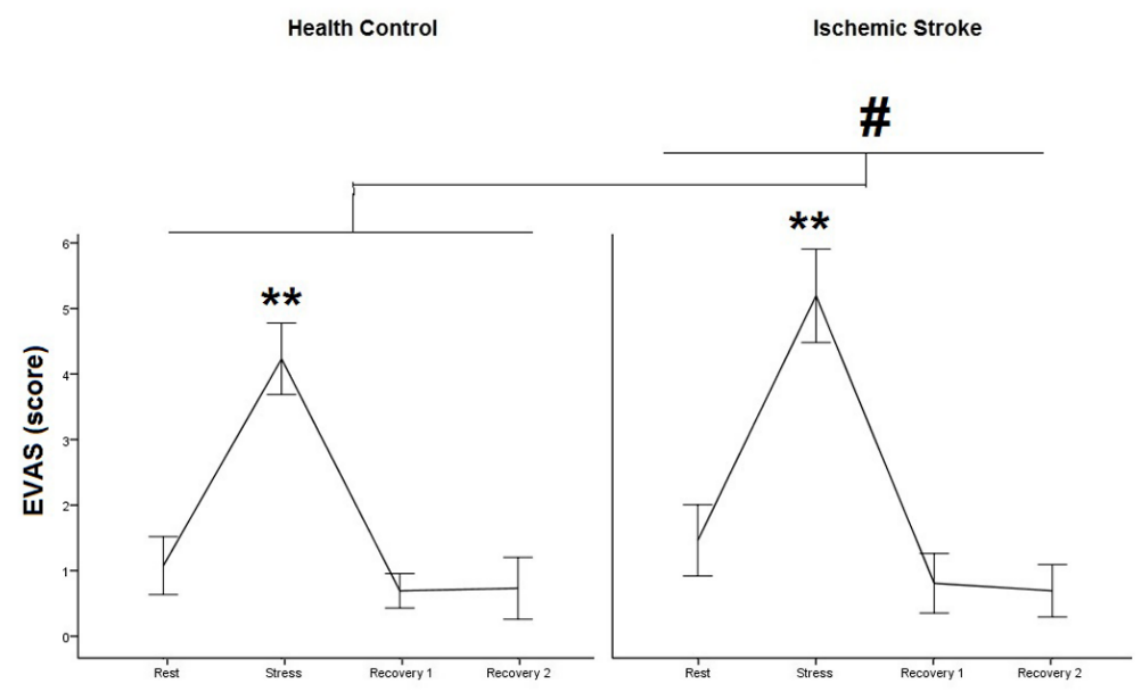

Figure 1: the plots show the mean and $95 \% \mathrm{Cl}$ of EVAS in the four times of test. ${ }^{* *}: p$-value $<0.000$ between stress and rest time per groups. \#: $p$-value $<0.05$ between two groups.

\section{Salivary cortisol}


Figure 2 shows the salivary cortisol level at four times of assessment in both groups. The results of Two-way mixed model ANOVA test showed that the interaction effect of time and group was significant ( F 5.09, P-value $<0.008$ ). It means that healthy individual's salivary cortisol levels significantly increased after the induced stress and it remained high during the first 20-minutes recovery time, but it began to decrease to the baseline levels during the second recovery time. While, unlike healthy controls, the salivary cortisol level of stroke patients decreased after stress, but this change was not statistically significant (figure 2).

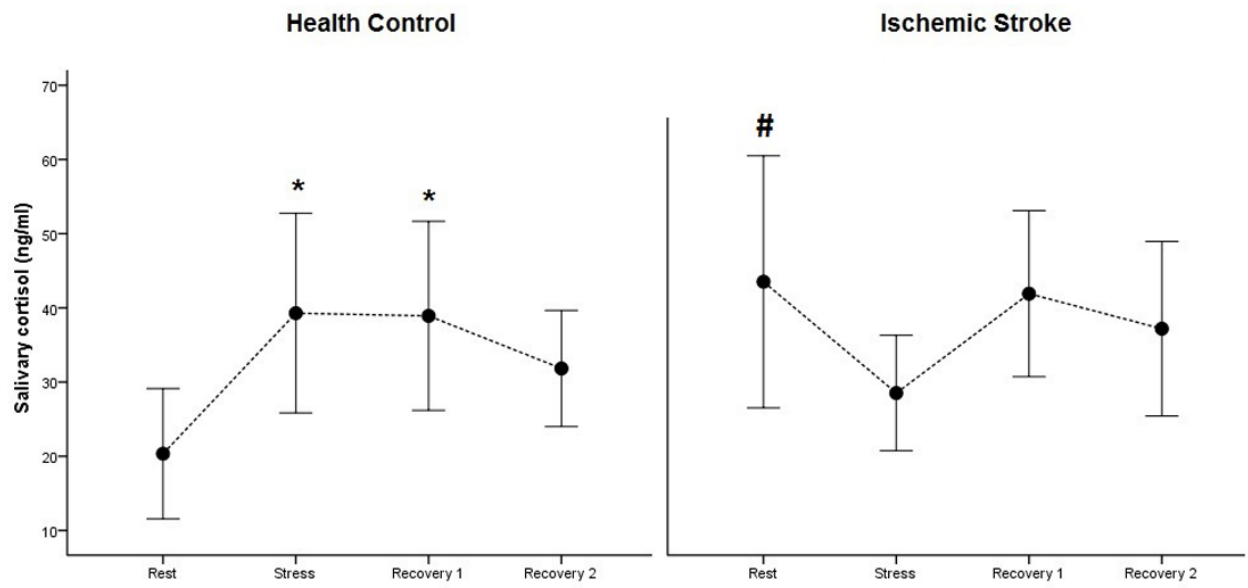

Figure 2: the plots show the mean and $95 \% \mathrm{Cl}$ of salivary cortisol at four times of measurement and in two groups (Healthy control and ischemic stroke groups). *: significant difference between the rest and other conditions. \#: significant difference between two groups in the rest.

\section{The features of HRV}

As shown in Figure 3, the number of heart beats per minute increased after the induced stress. As a result, the R-R intervals reduced in both groups, but these changes were significantly more in stroke patients $(F=21.97$, P-value $<0.001)$. After stress and during recovery time, the $R-R$ intervals restored to the baseline level.

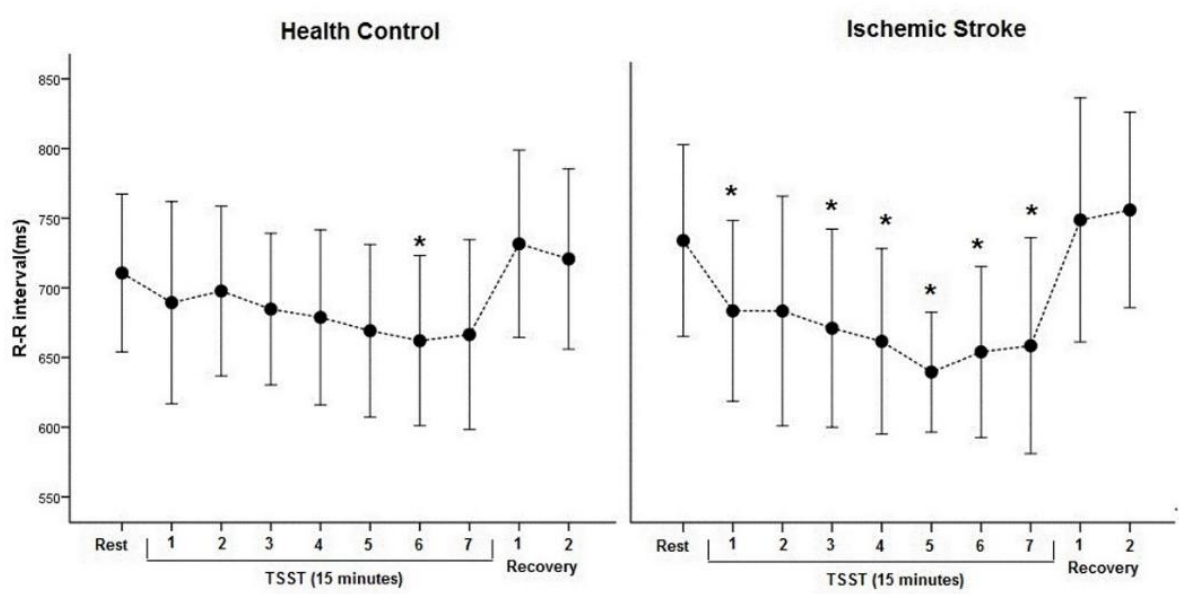

Figure 3: the plots show the mean and $95 \% \mathrm{Cl}$ of R-R interval of HRV in the repeated measurements in the rest, stress and recovery time. *: significant difference between the rest and other conditions.

Besides that, two-way mixed model ANOVA showed no significant difference in SD of $\mathrm{R}-\mathrm{R}$ interval of HRV in the repeated measurements at the rest, stress and recovery times between two groups (figure 4). As shown in figure 4, the diversity of SD of R-R interval was more between the stroke patients than the healthy controls, and the same finding was 
obtained for the SD1 of Poincare plot of HRV in the repeated measurements in the rest, stress and recovery time (figure 5).

Health Control

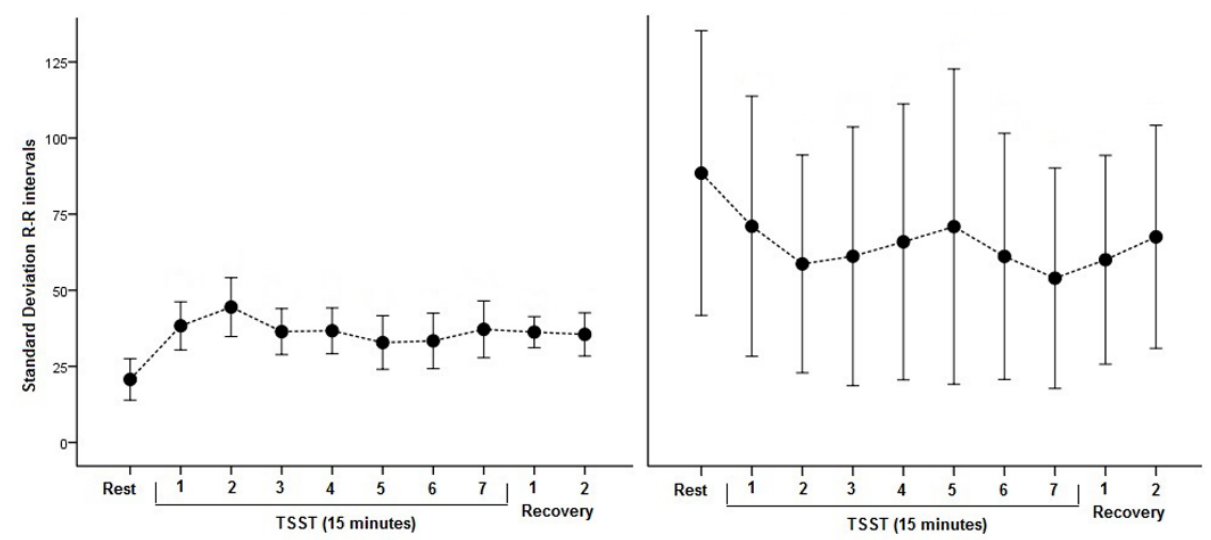

Figure 4: the plots show the mean and $95 \% \mathrm{Cl}$ of $\mathrm{SD}$ of $\mathrm{R}-\mathrm{R}$ interval of HRV in the repeated measurements in the rest, stress and recovery condition.

Health Control

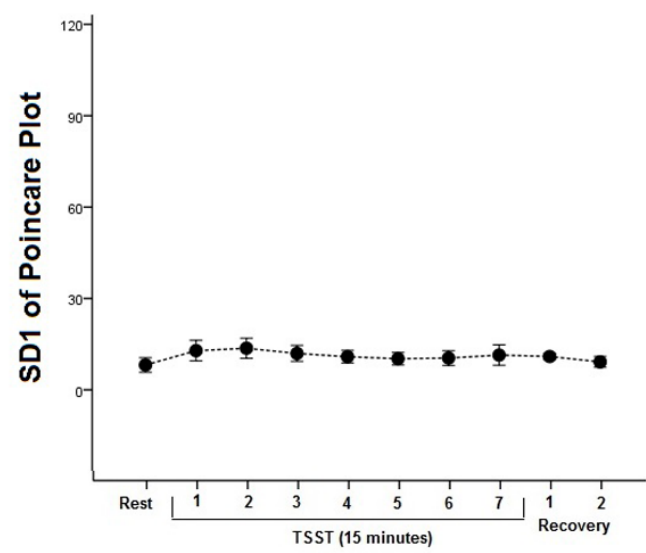

Ischemic Stroke

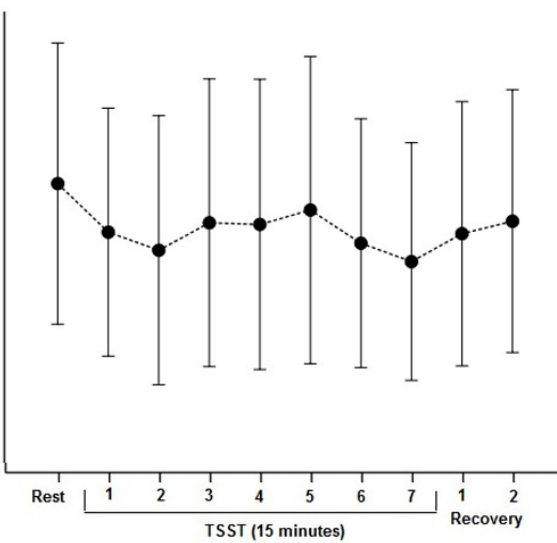

Figure 5: the plots show the mean and $95 \% \mathrm{Cl}$ of SD1 of Poincare plot of HRV in the repeated measurements in the rest, stress and recovery condition.

In the analysis of the relative power frequency of HRV, two-way mixed model ANOVA showed that the interaction effect of time and group was significant $(F=2.04, P$-value $<0.05)$. as shown in figure 6, the relative low frequency (LF) of HRV increased in both groups during the stress phase and came back to the baseline after recovery time, but, totally, the relative low frequency of HRV was significantly lower in the stroke group (P-value $<0.005)$. The changes of the relative low frequency of HRV during the stress phase and recovery time were more significant in the healthy individuals so that there was a statistically significant difference in the relative low frequency of HRV in healthy individuals between the second recovery time and the second two minutes of stress phase ( $P$-value $<0.05)$. The difference in the relative low frequency of HRV between the fifth two minutes of stress phase and the rest time in the stroke group was also significant (P-value $<0.05)$.

The relative high frequency (HF) of HRV decreased significantly in both groups sequentially $(F=3.17$, P-value $<0.05)$ and there was no significant difference between stroke patients and healthy controls (figure 7). There were no significant differences in the LF/HF and relative power of VLF between two groups and between different phases of the protocol. 
As shown in figure 8 , there was no difference in the alpha 1 DFA between rest time, stress phase and recovery time, but there was a significant difference in alpha 1 DFA measures between stroke group and healthy controls group (P-value $<0.05)$. Also, there was no difference in spectral and sample entropy measures between different times and different groups.
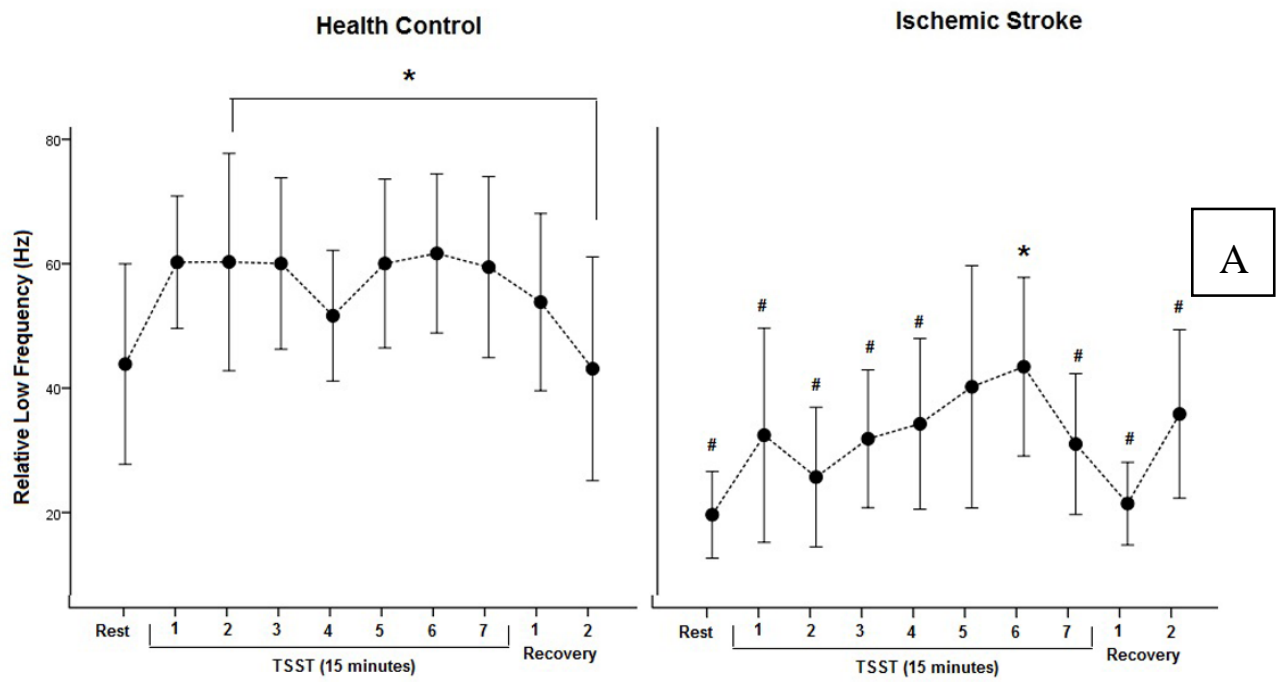

Health Control

Ischemic Stroke
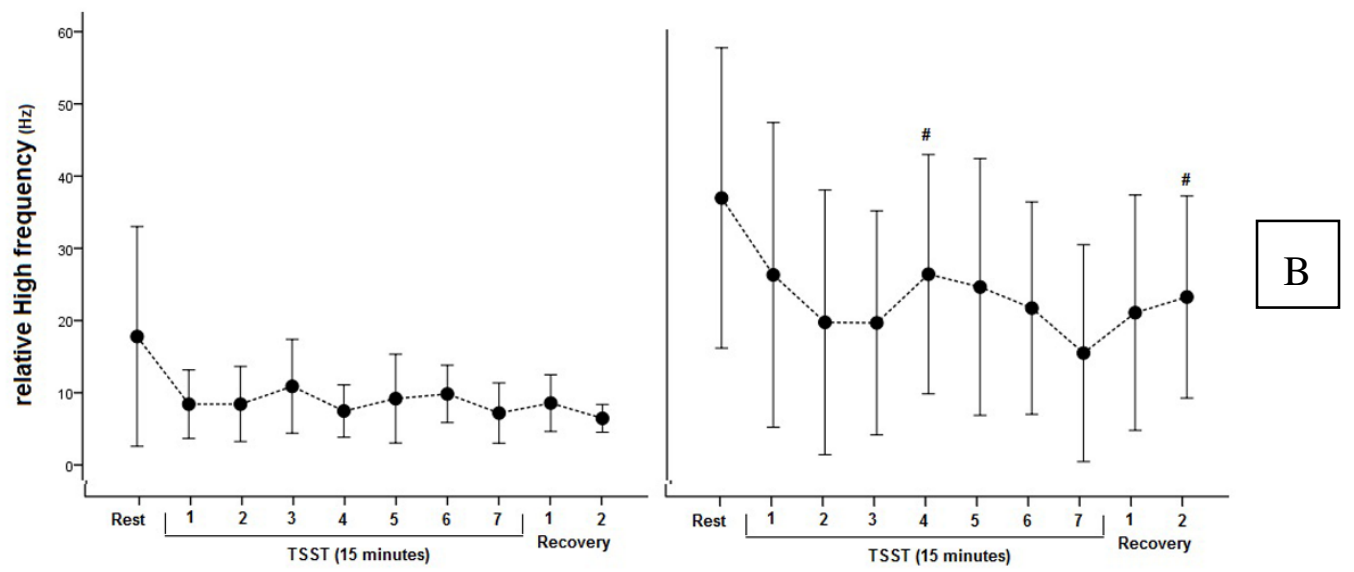

Figure 6: the plots show the mean and $95 \% \mathrm{Cl}$ of relative low frequency of HRV $(\mathrm{A})$ and high frequency of HRV (B) in the repeated measurements in the rest, stress and recovery condition. *: significant difference between conditions within group. \#: significant difference between two groups in the same condition. 


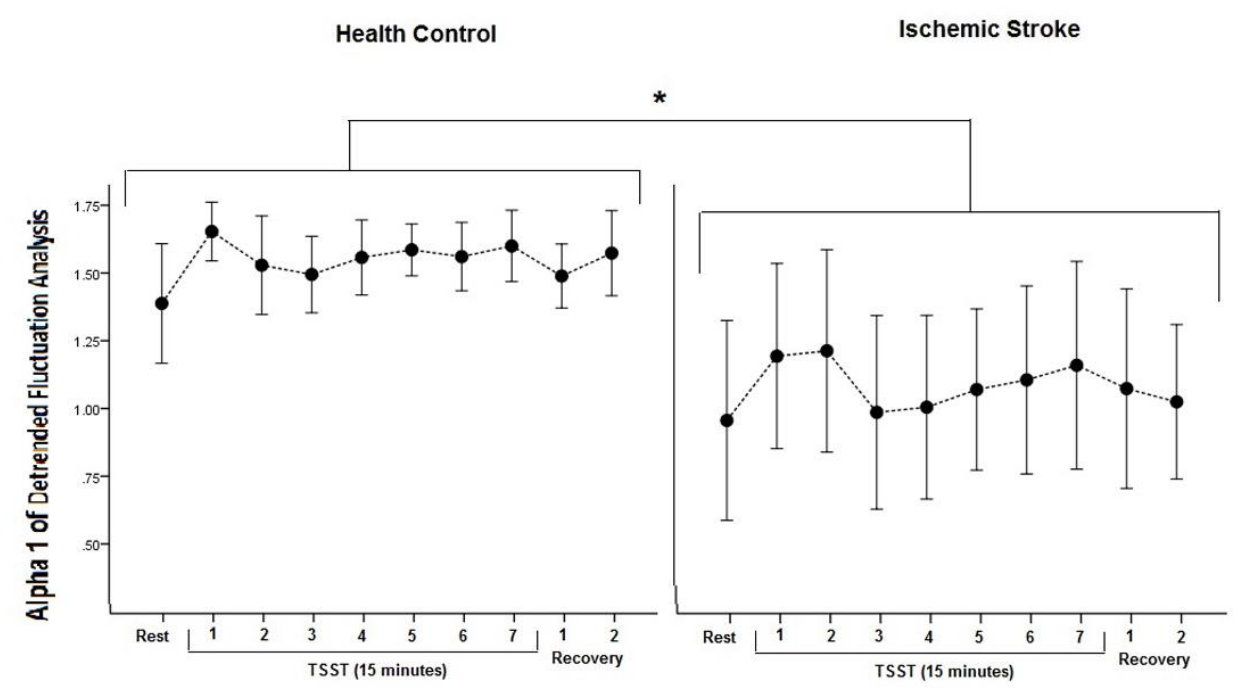

Figure 7: the plots show the mean and $95 \% \mathrm{Cl}$ of alpha 1 of DFA in the repeated measurements in the rest, stress and recovery condition. *: significant difference between within group

\section{DISCUSSION}

In the present study, in order to assess the stress system response in the post-stroke patients, TSST protocol was used. In both post-stroke and HC groups, the anxiety significantly increased after TSST based on EVAS score. Then, it was returned to baseline after recovery. The baseline comparison showed that stroke patients significantly had higher trait anxiety score. A recent systematic review involving 39 cohorts consist of 4,706 stroke patients showed that $24 \%$ of them had anxiety symptoms and $18 \%$ had an anxiety disorder in the first 5 years after stroke ${ }^{28}$. On the other hand the anxiety is a complication due to chronic stress ${ }^{16}$. Then there is a close chain of stroke, stressful events and mood disorder might be suggested.

\section{HPA axis assessment}

The change of salivary cortisol is the marker of HPA activity ${ }^{16}$. Basically, the resting salivary cortisol levels and anxiety score were higher in post-stroke patients than in healthy controls. After inducing stress, salivary cortisol levels raised in healthy controls and maintained after the first twenty minutes of recovery, while salivary cortisol tended to decrease non-significantly after stress in post-stroke patients. Some recent studies showed that cortisol levels in post-stroke patients are higher than healthy individuals ${ }^{22,29}$. Our finding supports the previous studies. Also, we found that anxiety level at the resting time and EVAS score after acute stress were higher in post-stroke patients than in healthy controls, which is probably due to the underlying ischemic stroke mechanism ${ }^{30}$. In fact, the higher level of anxiety at the resting time and higher EVAS score after stress could be a precursor for the higher level of salivary cortisol. Previous studies showed that the increase of cortisol after stress has negative correlation with baseline cortisol ${ }^{31,32}$. It is noticeable that, in our study, we also pointed out that the patients with chronic stroke responded to the acute stress with salivary cortisol decrease. This is also consistent with a previous study which showed that the cortisol level decreases after an acute stress in chronic stroke patients ${ }^{33}$. Then this study confirmed that chronic stroke patients exposed to chronic stress that showed no increase of cortisol after acute stress. Chronic stress increases the anxiety behavior and depression and decreases the production of brain-derived neurotrophic factor (BDNF) even in the offspring ${ }^{34}$. It is suggested to measure the level of BDNF that is a pathophysiological predictor for depression ${ }^{35}$. 


\section{SAM axis assessment}

The HRV features are the markers of SAM activity ${ }^{36}$. The results showed that the heart rate increased after acute stress and returned to the resting level during recovery time in both groups. The decrease of $R-R$ internal as a marker of heart rate during the stress was more significant in the post-stroke group. After acute stress, the LF power of HRV significantly elevated in both groups and then returned to the resting level after recovery which means that the sympathetic tone increased in both groups after acute stress. Basically, the LF power was significantly lower and HF power was significantly higher in the post-stroke group than healthy controls which means that the parasympathetic tone increased in post-stroke patients. This finding is in line with the previous findings confirming that in post-stroke patients parasympathetic overactivity occurrs ${ }^{37}$. Another important finding of our study was that SD of RR intervals, SD1 and SD2 of Poincaré Plot values, which both measure HRV volatilities, were very discrepant between post-stroke patients after stress, and there was less discrepancy in these values between healthy groups. This finding might be due to the different impacts of different locations of ischemic stroke on HRV ${ }^{38}$. Besides that, the non- linear features of HRV, like SD of Poincare, alpha 1 DFA, sample entropy in time domain and spectral entropy in the frequency domain (SpeEn), were not significantly different after and before acute stress in both post-stroke patients and healthy controls, despite of previous studies which showed that acute stress causes a decrease in the heart rate variability in the young voluntaries ${ }^{36}$.This mismatch between our finding and previous studies is probably contributable to the higher average age of participants in our study than previous studies, considering these facts that the HRV decreases in old ages ${ }^{39}$ and the brain functions and physiologic responses in older ages are diminished, so we should use modified HRV indices for different ages ${ }^{40}$. It is also notable that, in consistence with previous studies, we found significant lower mean values of alpha 1 of DFA in the post-stroke group at the resting time, which means that stroke causes a decrease in HRV ${ }^{41}$, 42

Many studies investigated cardiac complications and heart rate variability changes after stroke ${ }^{42-45}$. For example, in an earlier study, Fyfe-Johnson et al. found that the lower HF is correlated with the higher risk of stroke in adults ${ }^{46}$. In some other studies, it is shown that the patients with ischemic stroke had Autonomic Nervous System (ANS) dysfunction and decreased HRV compared to the healthy controls ${ }^{19,41}$, and this decreased HRV increases the stroke severity and risk of mortality ${ }^{47}$. As it was predictable, we found that LF of HRV significantly increased after stress and HF of HRV decreased at the same time in both groups, which can be interpreted as the increase of sympathetic tone due to stress ${ }^{48}$.

Our study has some limitations. First of all, we only enrolled patients with chronic ischemic stroke because the patients in the acute phase of stroke cannot participate in TSST protocol. The second limitation of our study was our limited sample size. So, we recommend that the future studies evaluate the physiological responses of acute stroke patients to the acute stress in the larger sample sizes.

\section{CONCLUSION}

In conclusion, in this study, we found that the post-stroke patients had different physiologic responses to the acute stress in comparison with healthy individuals. The post-stroke patients showed the increase of SAM activity based on HRV features due to stress but not HPA activity based on cortisol level. The non-linear HRV features of patients were very different that might be due to the different impaired region of brain, and need future research. The parasympathetic tone was also higher in post-stroke patients than $\mathrm{HC}$ group. The baseline trait anxiety score and salivary cortisol of patients significantly higher than $\mathrm{HC}$ and the change of cortisol due to TSST did not happen significantly in patients. Thus, the patients had signs of chronic stress and exposed them to several impairments such as mood disorders. The measurement of BDNF protein or gene is suggested to confirm in term of molecular assessment. 
Funding: No financial grant support the study and it was done by personal cost of researchers. Acknowledgments: the authors thank for technical assistant of Dr Mohammad Saleh KhajeHosseini and Zahra Arab.

Conflicts of Interest: The authors declare no conflict of interest.

\section{REFERENCES}

1. Estol CJ, Esnaola y Rojas MM. Stroke in Argentina. Int J Stroke. 2010; 5(1):35-9. 10.1111/j.1747-4949.2009.00402.x

2. Pirzad Jahromi G, Shabanzadeh Pirsaraei A, Sadr SS, et al. Multipotent bone marrow stromal cell therapy promotes endogenous cell proliferation following ischemic stroke. Clin Exp Pharmacol Physiol. 2015; 42(11):1158-67.

3. Koton S, Schneider AL, Rosamond WD, et al. Stroke incidence and mortality trends in US communities, 1987 to 2011. JAMA. 2014; 312(3):259-68. 10.1001/jama.2014.7692

4. Feigin VL, Forouzanfar MH, Krishnamurthi R, et al. Global and regional burden of stroke during 1990-2010: findings from the Global Burden of Disease Study 2010. Lancet. 2014; 383(9913):245-54.

5. Hosseini AA, Sobhani-Rad D, Ghandehari K, et al. Frequency and clinical patterns of stroke in Iran - Systematic and critical review. BMC Neurol. 2010; 10:72. 10.1186/1471-2377-10-72

6. Daneshfard B, Izadi S, Shariat A, et al. Epidemiology of stroke in Shiraz, Iran. Iran J Neurol. 2015; 14(3):158-63.

7. Pirzad Jahromi G, Shabanzadeh Pirsaraei A, Mokhtari Hashtjini M, et al. Bone marrow-derived mesenchymal stem cell and simvastatin treatment leads to improved functional recovery and modified c-Fos expression levels in the brain following ischemic stroke. Iran J Basic Med Sci. 2018 Oct;21(10):1004-1012.

8. Pirzad Jahromi G, Seidi S, Sadr SS, et al. Therapeutic effects of a combinatorial treatment of simvastatin and bone marrow stromal cells on experimental embolic stroke. Basic Clin Pharmacol Toxicol. 2012; 110(6):487-93.

9. Ferro JM, Caeiro L, Figueira ML. Neuropsychiatric sequelae of stroke. Nat Rev Neurol. 2016; 12(5):269-80. 10.1038/nrneurol.2016.46

10. Mitchell AJ, Sheth B, Gill J, et al. Prevalence and predictors of post-stroke mood disorders: A meta-analysis and meta-regression of depression, anxiety and adjustment disorder. Gen Hosp Psychiatry. 2017; 47:48-60. 10.1016/j.genhosppsych.2017.04.001

11. Carota A, Bogousslavsky J. Mood disorders after stroke. Front Neurol Neurosci. 2012; 30:70-4. $10.1159 / 000333413$

12. Bao AM, Meynen G, Swaab DF. The stress system in depression and neurodegeneration: focus on the human hypothalamus. Brain Res Rev. 2008; 57(2):531-53. 10.1016/j.brainresrev.2007.04.005

13. Caspi A, Sugden K, Moffitt TE, et al. Influence of life stress on depression: moderation by a polymorphism in the 5-HTT gene. Science. 2003; 301(5631):386-9. 10.1126/science.1083968

14. Kessler RC. The effects of stressful life events on depression. Annu Rev Psychol. 1997; 48:191-214. 10.1146/annurev.psych.48.1.191

15. Dalooei JR, Sahraei $\mathrm{H}$, Meftahi GH, et al. Temporary amygdala inhibition reduces stress effects in female mice. J Adv Res. 2016; 7(5):643-9.

16. Yaribeygi $\mathrm{H}$, Panahi $\mathrm{Y}$, Sahraei $\mathrm{H}$, et al. The impact of stress on body function: A review. EXCLI J. 2017; 16:1057-72.

17. McEwen BS. Physiology and neurobiology of stress and adaptation: central role of the brain. Physiol Rev. 2007; 87(3):873-904. 
18. Ori Z, Monir G, Weiss J, et al. Heart rate variability. Frequency domain analysis. Cardiol Clin. 1992; 10(3):499-537.

19. Dutsch M, Burger M, Dorfler C, et al. Cardiovascular autonomic function in poststroke patients. Neurology. 2007; 69(24):2249-55. 10.1212/01.wnl.0000286946.06639.a7

20. Miller AH, Spencer RL, McEwen BS, et al. Depression, adrenal steroids, and the immune system. Ann Med. 1993; 25(5):481-7.

21. Mitchell AJ. Clinical implications of poststroke hypothalamo-pituitary adrenal axis dysfunction: A critical literature review. J Stroke Cerebrovasc Dis. 1997; 6(6):377-88.

22. Barugh AJ, Gray P, Shenkin SD, et al. Cortisol levels and the severity and outcomes of acute stroke: a systematic review. J Neurol. 2014; 261(3):533-45. 10.1007/s00415-013-7231-5

23. Kirschbaum C, Pirke KM, Hellhammer DH. The 'Trier Social Stress Test'--a tool for investigating psychobiological stress responses in a laboratory setting. Neuropsychobiology. 1993; 28(1-2):76-81. 10.1159/000119004

24. Billman GE. The LF/HF ratio does not accurately measure cardiac sympatho-vagal balance. Front Physiol. 2013; 4:26. 10.3389/fphys.2013.00026

25. Brennan M, Palaniswami M, Kamen P. Do existing measures of Poincare plot geometry reflect nonlinear features of heart rate variability? IEEE Trans Biomed Eng. 2001; 48(11):1342-7.

26. Perfetto JC, Ruiz A, Attellis CD, editors. Detrended Fluctuation Analysis (DFA) and R-R Interval variability: A new linear segmentation algorithm. 2006 Computers in Cardiology; 2006 17-20 Sept. 2006.

27. Subha DP, Joseph P, Acharya U R, et al. EEG Signal Analysis: A Survey. J Med Syst. 2010; 34(2):195-212. 10.1007/s10916-008-9231-z

28. Kim JS. Post-stroke Mood and Emotional Disturbances: Pharmacological Therapy Based on Mechanisms. J of Stroke. 2016; 18(3):244-55. 10.5853/jos.2016.01144

29. Ben Assayag E, Tene O, Korczyn AD, et al. High hair cortisol concentrations predict worse cognitive outcome after stroke: Results from the TABASCO prospective cohort study. Psychoneuroendocrinology. 2017; 82:133-9. 10.1016/j.psyneuen.2017.05.013

30. Campbell Burton CA, Murray J, Holmes J, et al. Frequency of anxiety after stroke: a systematic review and meta-analysis of observational studies. Int J Stroke. 2013; 8(7):545-59. 10.1111/j.1747-4949.2012.00906.x

31. Mohammadi Alireza, Asgar Emamgoli, Maryam Shirinkalam, et al. the persistent response to acute psychosocial stress Biomedical Signal Processing and Control. 2019, 71:1-9

32. Takahashi $\mathrm{T}$, Ikeda $\mathrm{K}$, Ishikawa M, et al. Anxiety, reactivity, and social stress-induced cortisol elevation in humans. Neuro Endocrinol Lett. 2005; 26(4):351-4.

33. Laures-Gore J, Heim CM, Hsu YS. Assessing cortisol reactivity to a linguistic task as a marker of stress in individuals with left-hemisphere stroke and aphasia. J Speech Lang Hear Res. 2007; 50(2):493-507. 10.1044/1092-4388(2007/034)

34. Aliabadi N, Sahraei H, Bahari Z, et al. Effect of prenatal immobilization stress on spatial memory, anxiety-like behavior and brain BDNF concentration in the F1 generation male mice. Sci J Kurdistan Uni of Med Sci. 2018; 23(3):73-84.

35. Lee B-H, Kim Y-K. The roles of BDNF in the pathophysiology of major depression and in antidepressant treatment. Psychiatry investigation. 2010; 7(4):231-5. 10.4306/pi.2010.7.4.231 
36. Castaldo R, Melillo $P$, Bracale $U$, et al. Acute mental stress assessment via short term HRV analysis in healthy adults: A systematic review with meta-analysis. Biomed Signal Process Control. 2015; 18(Supplement C):370-7. https://doi.org/10.1016/j.bspc.2015.02.012

37. Soros P, Hachinski V. Cardiovascular and neurological causes of sudden death after ischaemic stroke. Lancet Neurol. 2012; 11(2):179-88. 10.1016/S1474-4422(11)70291-5

38. Colivicchi F, Bassi A, Santini M, et al. Prognostic implications of right-sided insular damage, cardiac autonomic derangement, and arrhythmias after acute ischemic stroke. Stroke. 2005; 36(8):1710-5. 10.1161/01.STR.0000173400.19346.bd

39. Antelmi I, de Paula RS, Shinzato AR, et al. Influence of age, gender, body mass index, and functional capacity on heart rate variability in a cohort of subjects without heart disease. Am J Cardiol. 2004; 93(3):381-5. 10.1016/j.amjcard.2003.09.065

40. Voss $A$, Schroeder R, Heitmann A, et al. Short-term heart rate variability--influence of gender and age in healthy subjects. PLoS One. 2015; 10(3):e0118308. 10.1371/journal.pone.0118308

41. Xiong L, Leung $\mathrm{HH}$, Chen $\mathrm{XY}$, et al. Comprehensive assessment for autonomic dysfunction in different phases after ischemic stroke. Int J Stroke. 2013; 8(8):645-51. 10.1111/j.1747-4949.2012.00829.x

42. Guan L, Collet JP, Mazowita G, et al. Autonomic Nervous System and Stress to Predict Secondary Ischemic Events after Transient Ischemic Attack or Minor Stroke: Possible Implications of Heart Rate Variability. Front Neurol. 2018; 9:90. 10.3389/fneur.2018.00090

43. Naver HK, Blomstrand C, Wallin BG. Reduced heart rate variability after right-sided stroke. Stroke. 1996; 27(2):247-51.

44. Korpelainen JT, Sotaniemi KA, Huikuri HV, et al. Abnormal heart rate variability as a manifestation of autonomic dysfunction in hemispheric brain infarction. Stroke. 1996; 27(11):2059-63. 45. Chen Z, Venkat $P$, Seyfried D, et al. Brain-Heart Interaction: Cardiac Complications After Stroke. Circ Res. 2017; 121(4):451-68. 10.1161/CIRCRESAHA.117.311170

46. Fyfe-Johnson AL, Muller CJ, Alonso A, et al. Heart Rate Variability and Incident Stroke: The Atherosclerosis Risk in Communities Study. Stroke. 2016; 47(6):1452-8. 10.1161/STROKEAHA.116.012662

47. Graff $B$, Gasecki $D$, Rojek $A$, et al. Heart rate variability and functional outcome in ischemic stroke: a multiparameter approach. J Hypertens. 2013; 31(8):1629-36. 10.1097/HJH.0b013e328361e48b

48. Goldstein DS. The autonomic nervous system in health and disease. New York: M. Dekker; 2001. xii, 618 p. p.

(C 2018 by the authors. Submitted for possible open access publication under the terms and conditions of the Creative Commons Attribution (CC BY NC) license (https://creativecommons.org/licenses/by-nc/4.0/). 